\title{
PANORAMAS METODOLÓGICOS EM REABILITAÇÃO NEUROPSICOLÓGICA NO BRASIL: DA TEORIA À PRÁTICA
}

\author{
Lucas Emmanuel Lopes e Santos ${ }^{{ }^{*}}$, Flávia da Cunha Pereira ${ }^{2}$ \& Cecília Souza Oliveira ${ }^{3}$
}

\begin{abstract}
RESUMO
SANTOS, L. E. L., PEREIRA, F. C., OLIVEIRA C. S. Panoramas Metodológicos Em Reabilitação Neuropsicológica no Brasil: da Teoria à Prática. Perpectivas Online: Humanas \& Sociais Aplicadas, v.7, n.20, p.1-10, 2017.

A neuropsicologia é a ciência que trata das relações entre o cérebro e o comportamento, a partir da investigação de alterações cognitivas e comportamentais associadas às lesões cerebrais e distúrbios neurológicos e/ou psiquiátricos. Dentre as diversas propostas de intervenção que constituem o domínio da neuropsicologia, a reabilitação neuropsicológica $(\mathrm{RN})$ tem a finalidade de capacitar pacientes e familiares a conviver, reduzir ou superar os déficits cognitivos resultantes de lesão ou transtorno neurológico. O presente estudo teve como objetivo realizar uma revisão sistemática da literatura e traçar um panorama do desenvolvimento teórico e prático da

"reabilitação neuropsicológica". A amostra final resultou em um total de 22 artigos científicos, abrangendo o período de 2000 a 2016. Evidenciou-se que: a) o maior número de publicações sobre o tema se deu no ano de 2011, representando $18,2 \%$ da produção total; b) a grande maioria dos trabalhos publicados se refere à estudos de caso, totalizando $36 \%$ da produção; c) apenas $18 \%$ das publicações apresenta metodologia detalhada do plano de reabilitação neuropsicológica utilizado. Os resultados nos permitem concluir que estudos acerca dos benefícios da reabilitação neuropsicológica ainda são escassos no Brasil.
\end{abstract} $\mathrm{RN}$ no Brasil. A pesquisa foi realizada na base de dados LILACS, utilizando-se o unitermo

Palavras-chave: Neuropsicologia; Reabilitação neuropsicológica; Comprometimento neurológico; Produção científica. 


\begin{abstract}
Neuropsychology is the science that deals with the relations between the brain and behavior, from the investigation of cognitive and behavioral changes associated with brain injuries and neurological and/or psychiatric disorders. Among the various intervention proposals that constitute the domain of neuropsychology, neuropsychological rehabilitation (NR) has the purpose of enabling patients and families to coexist, reduce or overcome the cognitive deficits resulting from neurological injury or disorder. The present study aimed to carry out a systematic review of the literature and outline the theoretical and practical

keyword "neuropsychological rehabilitation". The final sample resulted in a total of 22 scientific articles, covering the period from 2000 to 2016 . It was evidenced that: a) the largest number of publications on the subject occurred in the year 2011 , accounting for $18.2 \%$ of total production; $b$ ) the vast majority of published works refer to case studies, totaling $36 \%$ of production; c) only $18 \%$ of the publications present a detailed methodology of the neuropsychological rehabilitation plan used. The results allow us to conclude that studies about the benefits of neuropsychological rehabilitation are still scarce in Brazil.
\end{abstract} development of NR in Brazil. The research was performed on LILACS database, using the

Keywords: Neuropsychology; Neuropsychological rehabilitation; Neurological impairment; Scientific production.

${ }^{1}$ Graduando em Psicologia da Universidade Federal Fluminense - UFF- Rua José do Patrocínio, 71 - Centro, Campos dos Goytacazes - RJ, CEP: 28010-385, Brasil;

${ }^{2}$ Mestranda em Cognição e Linguagem Universidade Estadual do Norte Fluminense Darcy Ribeiro - UENF- Avenida Alberto Lamego, 2000 - Parque California, Campos dos Goytacazes - RJ,CEP: 28013-602, Brasil;

${ }^{3}$ Docente do curso de Graduação em Psicologia da Universidade Federal Fluminense - UFF- Rua José do Patrocínio, 71 - Centro, Campos dos Goytacazes - RJ, CEP: 28010-385, Brasil.

(*)e-mail: lucaselopes16@gmail.com

Data de chegada: 16/08/2017 Aceito para publicação: 06/12/2017.

Persp. online: hum. \& sociais aplicada., Campos dos Goytacazes, 20 (7), 1-10, 2017

seer.perspectivasonline.com.br 


\section{INTRODUÇÃO}

\subsection{Neuropsicologia: ciência e prática}

A psicologia enquanto ciência plural e interdisciplinar tem como objeto de estudo os fenômenos do comportamento humano, tendo como pressupostos os fatores biopsicossociais que fundamentam sua constituição. Dentre os diversos ramos de investigação psicológica, a neuropsicologia surge apoiada por contribuições do campo das neurociências, tais como a neuroanatomia, neurofisiologia e neurofarmacologia, e também de áreas já consolidadas da psicologia, como a psicometria, psicologia experimental e psicologia cognitiva (HAMDAN, PEREIRA \& RIECHI, 2011).

As raízes da neuropsicologia remontam, para Riccio, Sullivan \& Cohen (2010), à Antiguidade. Um dos primeiros registros escritos sobre o estudo do cérebro é datado de 3500 a.C, e ficou conhecido como o "Papiro Cirúrgico de Edwin Smith". O documento, considerado o primeiro tratado de cirurgia, citava possíveis crises convulsivas e inaugurou as investigações sobre o encéfalo humano, além de colaborar para avanços significativos na compreensão de doenças como a epilepsia (MANCZAK, 2003; PINHEIRO, 2005). A partir do desenvolvimento da neurologia comportamental, na Grécia e Egito Antigos, tornou-se possível a investigação dos distúrbios de funções cognitivas superiores, tais como a linguagem e a percepção visual. Ainda segundo os autores, a neurologia comportamental parte do pressuposto de que o comportamento é, em última análise, dependente do funcionamento do sistema nervoso central. Nessa perspectiva, a neuropsicologia é o campo científico que trata das relações entre o cérebro e o comportamento, a partir da investigação de alterações cognitivas e comportamentais associadas às lesões cerebrais e distúrbios neurológicos e/ou psiquiátricos.

De acordo com Gindri et al. (2012), o estudo sistemático da relação entre lesões cerebrais e o comportamento se deu a partir da Segunda Guerra Mundial (1939-1945). Para os autores, a utilização de testes neuropsicológicos tornou-se de fundamental importância para o diagnóstico de comprometimentos cognitivos em soldados de guerra, além de servirem como uma ferramenta de avaliação dos avanços obtidos pelas intervenções neuropsicológicas. Nesse contexto, destaca-se o trabalho de Leonard Diller, que adotou um modelo holístico de reabilitação no qual, para o seu planejamento, considerava os aspectos emocionais e sociais causados pela lesão cerebral (GINDRI et al., 2012).

Os estudos realizados por Alexander Luria também foram de grande relevância no cenário da reabilitação neuropsicológica, especialmente no que se refere aos soldados com lesões cerebrais. Segundo Luria (1981), a neuropsicologia é um ramo da ciência que tem por objetivo específico a investigação da função de sistemas cerebrais individuais em formas complexas de atividade mental. De acordo com o autor, tal campo de estudo se tornou um instrumento que possibilita a revisão de nossos conceitos fundamentais sobre a estrutura interna de processos psicológicos e um fator fundamental que leva à criação de uma teoria da base cerebral da atividade mental humana.

Na compreensão de Hebben \& Milberg (2002), a psicologia clínica se fundamentou a partir das contribuições da psicologia da aprendizagem e cognição, psicologia do desenvolvimento, psicologia social e das tradições psicodinâmicas. Para os autores, a neuropsicologia clínica agrega a esse arcabouço teórico os paradigmas da biologia e da medicina, visando o entendimento dos processos psicopatológicos humanos.

Conforme Hamdan, Pereira \& Riechi (2011), pode-se observar que os principais empregos de tal área da psicologia são a avaliação e a reabilitação neuropsicológica. Os conhecimentos produzidos pela neuropsicologia têm um amplo emprego na investigação científica e na aplicação desses conhecimentos no campo profissional. A análise de tais constructos auxilia na busca de explicações sobre as expressões cognitivas e comportamentais decorrentes de alterações cerebrais. Na atuação profissional, a avaliação e a reabilitação neuropsicológica contribuem para a identificação, documentação e tratamento das alterações cognitivas e comportamentais presentes em diversas situações nas quais o sistema nervoso central é afetado.

Persp. online: hum. \& sociais aplicada., Campos dos Goytacazes, 20 (7), 1-10, 2017

seer.perspectivasonline.com.br 
Além disso, a aplicabilidade clínica permite que o neuropsicólogo esclareça para o paciente, seus familiares e a equipe de saúde a respeito das suas limitações e suas potencialidades cognitivas e comportamentais, tornando possível que, após o estabelecimento deste perfil, sejam realizados planos de reabilitação neuropsicológica mais eficazes.

\subsection{Avaliação neuropsicológica}

A avaliação neuropsicológica tem como principais objetivos contribuir para o diagnóstico diferencial, investigar se há ou não disfunção cognitiva e o nível de funcionamento em relação ao nível ocupacional, além de auxiliar na localização de alterações sutis, possibilitando identificar as disfunções ainda em estágios iniciais, de forma a propiciar um tratamento eficaz e o acompanhamento da evolução do paciente perante o tratamento proposto (MÄDER, 1996).

Na perspectiva de Riccio, Sullivan \& Cohen (2010), um dos objetivos iniciais da avaliação neuropsicológica é identificar ou descartar determinada patologia. Luria (1980), Riccio \& Reynolds (1998) (apud RICCIO et al., 2010) afirmam que a conceitualização de caso na avaliação neuropsicológica deve reunir informações que envolvam diferentes domínios comportamentais, e que possam refletir o funcionamento de sistemas neurológicos. Nesse sentido, a avaliação neuropsicológica tem como premissa a incorporação de todos os sistemas e estruturas que estejam relacionados ao funcionamento neurológico do indivíduo.

Em conformidade com Lezak, Howieson e Loring (2004), a avaliação neuropsicológica ocupa lugar central no que se refere aos cuidados com o indivíduo. Nesse sentido, a avaliação neuropsicológica pode fornecer aos membros de seu convívio familiar e social informações importantes relativas às suas capacidades e limitações. Tais informações incluem a capacidade de autocuidado, capacidade de seguir o tratamento proposto, reações às suas próprias limitações, adequação de sua avaliação de bens e dinheiro, dentre outras.

Segundo Hebben \& Milberg (2002), a avaliação neuropsicológica deve considerar tanto os aspectos quantitativos quanto qualitativos para a compreensão do perfil cognitivo dos pacientes. Além disso, os autores demonstram a aplicabilidade da avaliação neuropsicológica em pelo menos sete diferentes contextos, incluindo a medicina, o direito, a educação e em pesquisas científicas.

De acordo com Ramos \& Hamdan (2016), os recursos utilizados na avaliação neuropsicológica são diversos, sendo este um procedimento investigativo realizado mediante a entrevistas, observações, provas de rastreio e baterias de testes psicométricos que procuram identificar o rendimento cognitivo funcional, a partir do conhecimento de suas relações com o funcionamento cerebral, investigando deste modo, níveis de comprometimento das funções cognitivas em questão.

Para Cruz, Schewinsky \& Alves (2012) a avaliação neuropsicológica exige do profissional que atua na área não apenas uma consolidada fundamentação em psicologia clínica e familiaridade com a psicometria, mas também especialização e treinamento em contextos nos quais seja fundamental o conhecimento do sistema nervoso e de suas patologias. Tal modelo de avaliação possibilita a investigação de determinada função cognitiva para observar seu ajustamento ou comportamento, como por exemplo: memória, atenção, linguagem, funções executivas, raciocínio, motricidade e percepção, bem como alterações afetivas e de personalidade (HAMDAN et al., 2011).

É importante ressaltar que a meta da avaliação neuropsicológica não deve ser apenas para fins de diagnóstico, mas, nas palavras de Riccio et al. (2010), "para a identificação de pontos fortes e fracos individuais com a intenção de informar os esforços de reabilitação". Diante disso, o propósito da avaliação deve ser o de desenvolver planos de intervenção ou reabilitação neuropsicológicas que atendam as demandas particulares do indivíduo. 


\subsection{Reabilitação neuropsicológica}

Este ramo da neuropsicologia tem por objetivo capacitar pacientes e familiares a conviver, lidar, contornar, reduzir ou superar as deficiências cognitivas, emocionais e sociais, proporcionando deste modo uma melhora significativa na qualidade de vida dos sujeitos envolvidos (NOMURA et al., 2000, apud SIMON \& RIBEIRO, 2011). Segundo Fuentes, Malloy-Diniz, Camargo \& Cosenza (2014), os resultados obtidos em tal procedimento fazem do profissional de neuropsicologia uma espécie de agente de mudança, sendo necessário muito cuidado e cautela para obter resultados positivos ao longo do tratamento, além de aliar teoria e prática para que o processo seja bem-sucedido.

A seleção de práticas baseadas em evidências é, segundo Riccio, Sullivan \& Cohen (2010), um importante componente para o planejamento de uma reabilitação neuropsicológica eficaz. De acordo com Chambliss \& Ollendick (2001, apud RICCIO et al., 2010) o estabelecimento de um programa de reabilitação deve considerar evidências de pesquisa, conhecimentos clínicos e também a adequação dos instrumentos para o paciente. Além disso, deve-se considerar cada área-alvo separadamente, uma vez que a eficácia de uma intervenção pode variar de acordo com os pontos fortes e fracos do indivíduo (RICCIO et al., 2010).

Em estudo de revisão desenvolvido por Miotto et al. (2008) com o intuito de investigar técnicas de reabilitação neuropsicológica em idosos com queixas cognitivas ou com diagnóstico de transtorno cognitivo leve, foram identificados 8 artigos que descreveram as avaliações neuropsicológicas realizadas. Os autores identificaram que os principais achados se referem aos benefícios da utilização de técnicas de reabilitação neste público da terceira idade. Contudo, ainda são poucas as técnicas que são empregadas na clínica e que podem ser generalizadas para as atividades do dia a dia, o que poderia refletir em uma maior autonomia da pessoa idosa na realização das suas atividades. Em adição, Miotto et al. (2008) concluem que ainda são necessários mais estudos nesta área, principalmente considerando os prejuízos e potencialidades cognitivas do idoso, bem como a situação clínica e familiar que o mesmo se encontra.

Ainda em Fuentes et al. (2014), podemos constatar que as ações tomadas no processo de reabilitação neuropsicológica devem estar focadas nos ganhos funcionais do paciente em questão. Este tipo de prática centrada tem como passo inicial verificar, através de uma entrevista detalhada, quais são as principais queixas do paciente e de seus familiares e, a partir deste contexto, traçar um plano de reabilitação neuropsicológica condizente com o perfil clínico e cognitivo do mesmo. Deste modo, o papel central do profissional especialista em neuropsicologia é tentar diminuir os impactos que as dificuldades podem trazer à vida do indivíduo.

Segundo Cruz, Schewinsky \& Alves (2012), a reabilitação neuropsicológica considera também o tratamento através de psicoterapia, seja ela individual e/ou grupal e familiar. A individual visa a organização da rotina do paciente, buscando o planejamento de novas atividades e estratégias, além de suporte emocional para percepção do problema e melhora do controle comportamental. Por outro lado, a realizada em grupo tem foco no treino de habilidades sociais e na percepção de respostas não esperadas, ou seja, inadequadas ao planejamento proposto.

Esta área de pesquisa é recente no Brasil, considerando que a neuropsicologia foi regulamentada como uma especialização da psicologia no ano de 2004. Deste modo, a presente pesquisa tem por objetivo realizar uma revisão sistemática da literatura e traçar um panorama do desenvolvimento teórico e prático da reabilitação neuropsicológica no país. Além disso, pretende-se verificar a produção total de artigos nacionais sobre reabilitação neuropsicológica entre os anos 2000 a 2016, as revistas em que foram publicadas, o tamanho das amostras utilizadas nas publicações, além de fazer um levantamento da metodologia utilizada para o plano de reabilitação neuropsicológica nos trabalhos analisados.

\section{METODOLOGIA}

Realizou-se uma busca eletrônica na base de dados LILACS por artigos publicados no Brasil, entre os anos 2000 a 2016. A pesquisa foi limitada ao unitermo "reabilitação neuropsicológica" e a trabalhos 
escritos em português do Brasil. Primeiro fez-se o levantamento das publicações, seguido da leitura dos resumos e análise dos artigos conforme os objetivos deste trabalho. Em seguida, os dados foram categorizados de acordo com as seguintes variáveis: a) número de publicações por ano; b) revistas em que foram publicados; c) tipo de produção científica; d) região do país em que foram publicados; e) tamanho da amostra e f) metodologia utilizada para o plano de reabilitação neuropsicológica.

\subsection{Critérios de inclusão e exclusão de trabalhos}

Para este estudo de revisão foram excluídos os artigos não indexados, teses, dissertações, resenhas, livros, cartas aos editores e relatos de prática profissional.

\section{RESULTADOS E DISCUSSÃO}

Apesar da expressiva contribuição que a reabilitação neuropsicológica desempenha no tratamento e na diminuição dos sintomas resultantes de déficits cognitivos, a temática ainda tem sido pouco explorada nas revistas brasileiras indexadas. No período entre 2000 e 2016, de acordo com os critérios de inclusão e exclusão descritos na metodologia, verificou-se um total de 22 artigos. Como pode ser observado na Figura 1, o número de publicações por ano limitou-se a 1 ou 2 artigos, com exceção do ano de 2011, no qual foram encontradas 4 publicações sobre o tema.

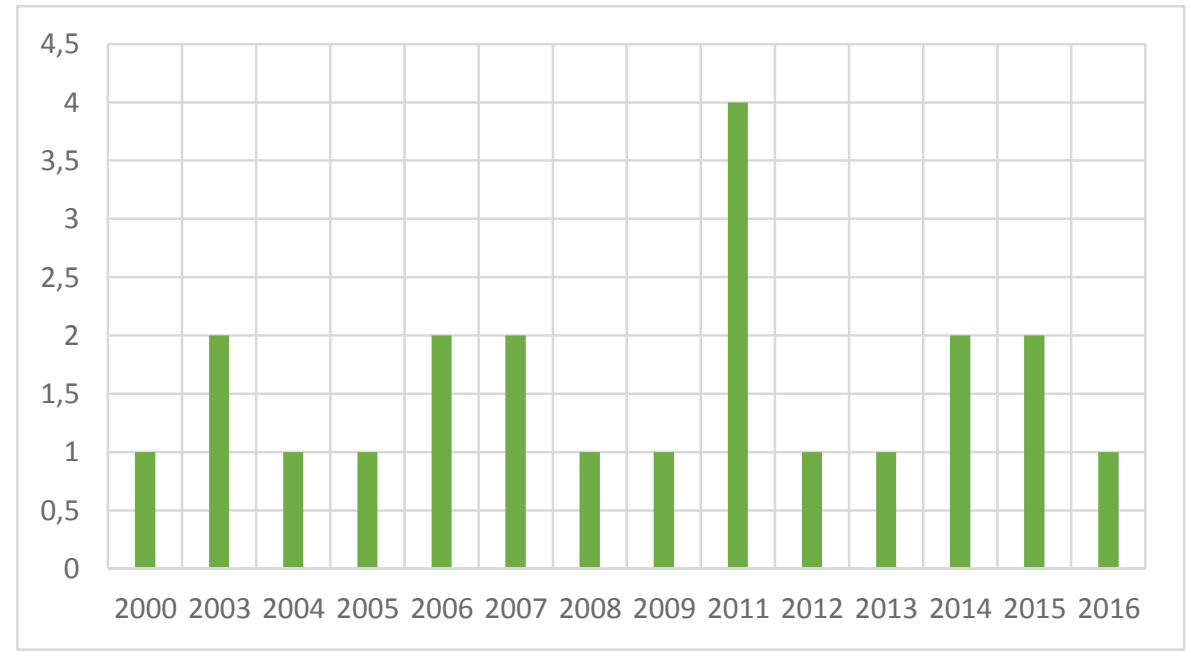

Figura 1: Total de artigos em português sobre reabilitação neuropsicológica com publicações entre 2000 e 2016.

O interesse na relação entre o cérebro e o comportamento humano é historicamente constituído por diferentes vertentes e áreas do conhecimento. Luria (1981) ressalta que o estudo sobre o funcionamento do cérebro como o órgão da atividade mental é uma tarefa complexa, que exige observações e contribuições de diversos campos da ciência, como a morfologia, fisiologia, psicologia e clínica médica.

Diante dos pressupostos da interdisciplinaridade como um caminho seguro para a investigação de aspectos tão complexos como a atividade mental, fica evidente que o apoio multiprofissional para o diagnóstico e tratamento de déficits cognitivos é fundamental na garantia de melhores resultados. Nessa perspectiva, é interessante notar que as revistas científicas brasileiras têm proporcionado uma investigação a partir de uma vertente multidisciplinar, como pode ser constatado na Figura 2. As revistas com maior número de publicações são de diferentes áreas do conhecimento, como psicologia $(\mathrm{n}=3)$, psiquiatria $(\mathrm{n}=3)$ e medicina física e reabilitação $(n=2)$. 


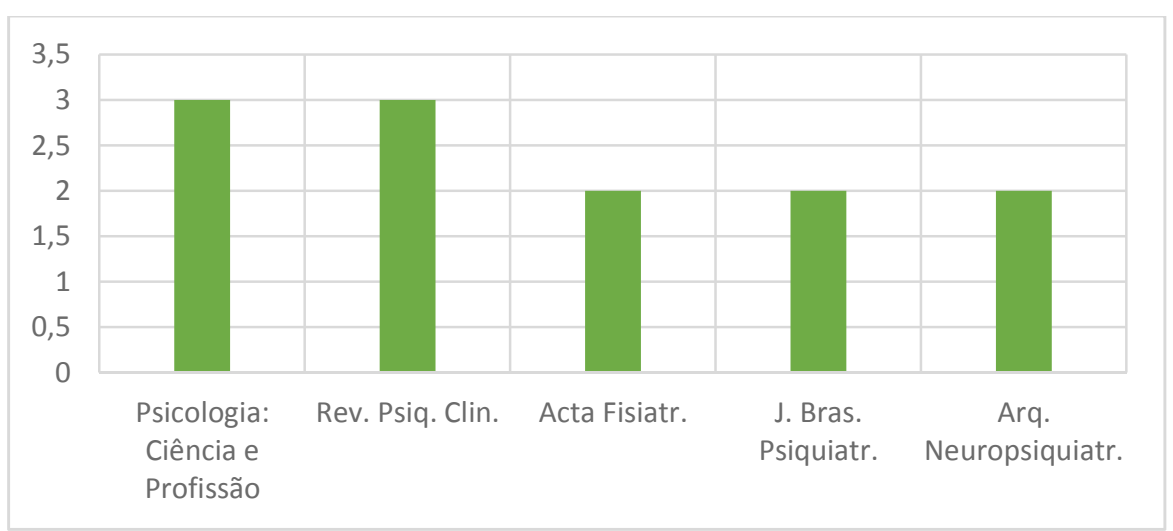

Figura 2: Revistas brasileiras com maior número de publicações sobre o tema.

A análise qualitativa dos artigos evidenciou dois tipos de produção científica predominantes nas revistas brasileiras: $36 \%$ dos trabalhos se refere à estudos de caso $(n=8)$, seguido de 7 artigos caracterizados como revisões de literatura, representando $31,8 \%$ das produções sobre a temática da reabilitação neuropsicológica. Vale ressaltar que embora os estudos de casos sejam relevantes para esclarecer a respeito da condição clínica do paciente aliado ao tipo de técnica de reabilitação empregada, muitas vezes o sucesso e a limitação do método restringem-se àquele paciente específico, não sendo possível, desta forma, a generalização dos resultados com a utilização das mesmas atividades em um grupo maior de pacientes.

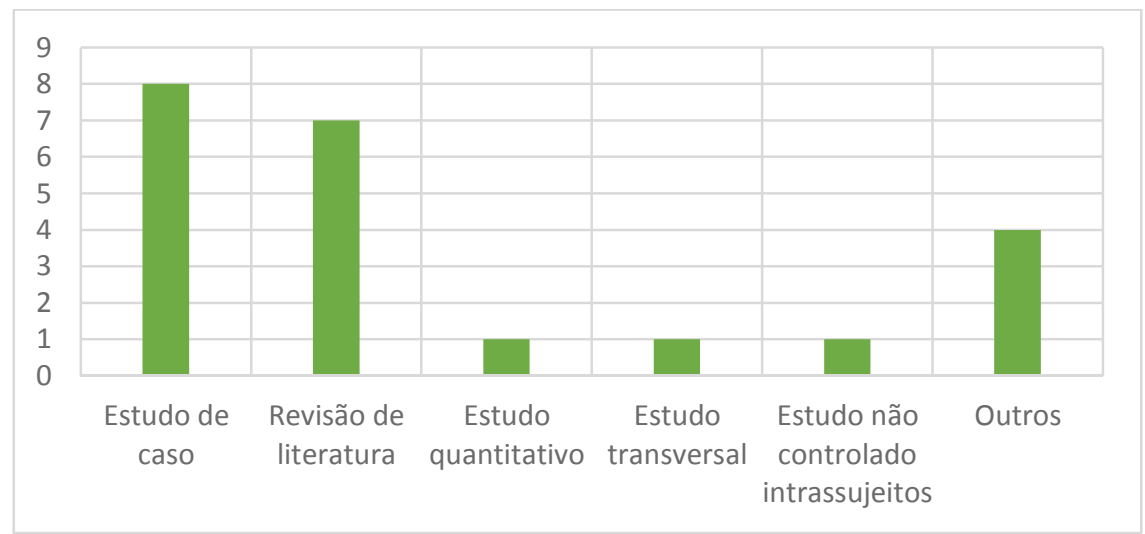

Figura 3: Tipos de publicações científicas sobre reabilitação neuropsicológica em revistas brasileiras.

Com relação à região do Brasil em que os trabalhos foram publicados, constatou-se que a grande maioria dos artigos $(n=13)$ vem da região Sudeste, totalizando $59 \%$ da amostra analisada. Como demonstrado na Figura 4, a região Sul foi responsável pela produção de 6 artigos, seguidos de 2 da região Nordeste e 1 da região Centro-Oeste. Vale ressaltar a ausência de produções científicas sobre a temática na região Norte do país, dentro do período estabelecido e conforme os critérios metodológicos deste estudo. Destaca-se que a concentração de pesquisas com esta temática na região Sudeste deve-se ao fato de que os principais centros neurológicos do país estão localizados justamente nestas áreas. Em adição, as especializações em neuropsicologia e em reabilitação neuropsicológica também são, em sua grande maioria, na região Sudeste, dificultando, deste modo, que os profissionais psicólogos de outras regiões tenham acesso a atualizações teóricas sobre esta temática. 


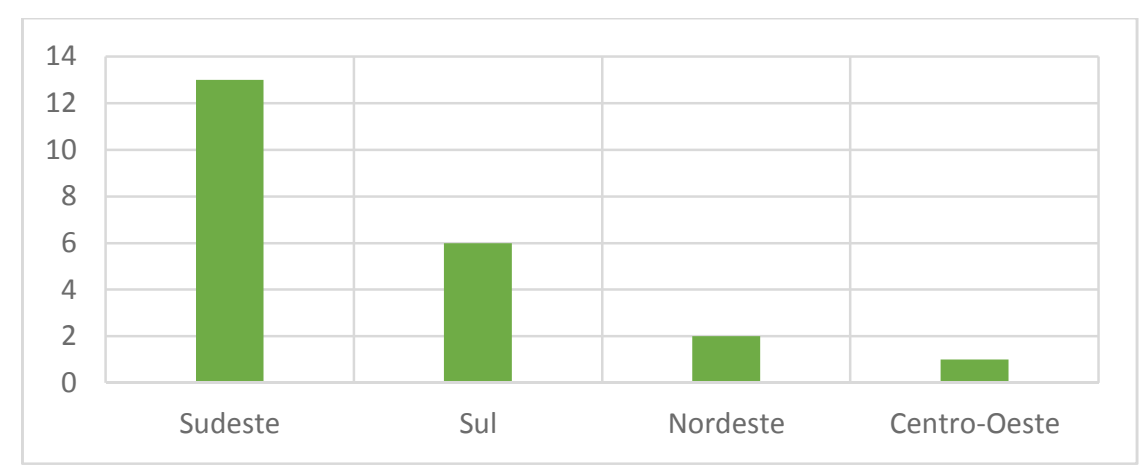

Figura 4: Região do Brasil em que os trabalhos foram publicados.

A Figura 5 apresenta o total da amostra de sujeitos nos artigos analisados. Foi demonstrado que em $50 \%$ das publicações $(\mathrm{n}=11)$ o número de participantes não foi evidenciado. Uma hipótese para este resultado é que grande parte dos trabalhos se tratava de estudos de revisão. Além disso, $45 \%$ dos artigos $(\mathrm{n}=$ 10) contou com menos de 100 sujeitos na amostra total.

Apenas 1 artigo teve a amostra investigada com um número superior a 100 sujeitos. De acordo com Serapioni (2000), apesar dos estudos qualitativos apresentarem validade interna, uma vez que se concentram na investigação de particularidades e características específicas de um grupo, são deficitários na sua capacidade de generalizar os resultados para uma população mais ampla.

Deste modo, seriam necessários mais estudos, em nosso país, envolvendo um número maior de sujeitos na amostra analisada a fim de que os resultados encontrados possam, de fato, refletir as contribuições da reabilitação neuropsicológica, possibilitando, assim, a análise de nuances características da população brasileira.

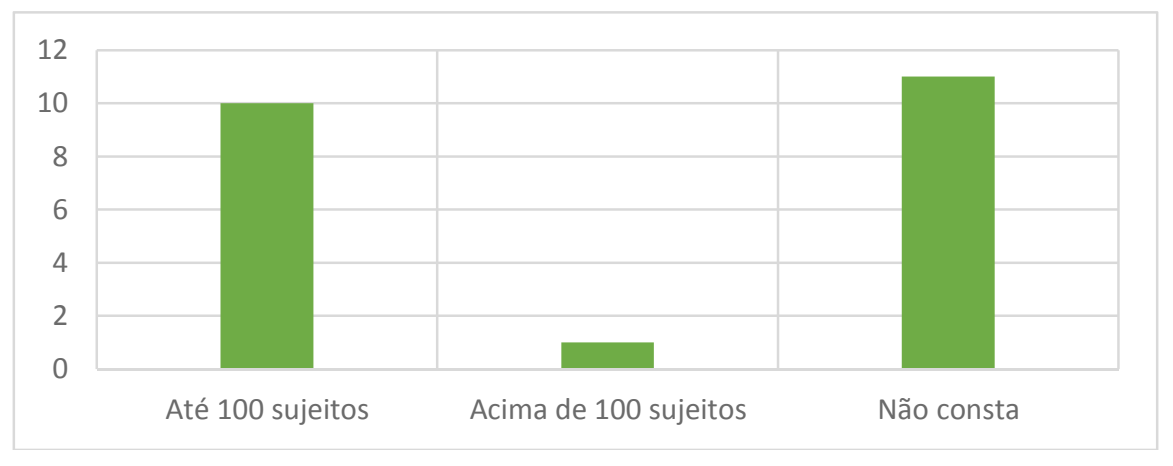

Figura 5: Total da amostra de sujeitos nos artigos analisados.

Conforme afirmam Hamdan et al. (2011), a reabilitação neuropsicológica se constitui como um processo que busca tratar ou aliviar deficiências cognitivas resultantes de uma lesão neurológica e contam, para isso, com a cooperação de profissionais da saúde, familiares e membros da comunidade. Wilson (apud HAMDAN et. al, 2011) ressalta que o objetivo da reabilitação é capacitar pacientes e familiares a conviver, lidar, contornar, reduzir ou superar tais deficiências cognitivas.

Para uma avaliação mais precisa e eficaz, faz-se necessária a utilização de instrumentos como testes neuropsicológicos, entrevistas e observações clínicas. Diante disso, a análise dos artigos publicados constatou que apenas $18 \%$ dos trabalhos $(n=4)$ demonstrou de forma clara a metodologia utilizada para $o$ plano de reabilitação neuropsicológica, como pode ser observado na Figura 6. 


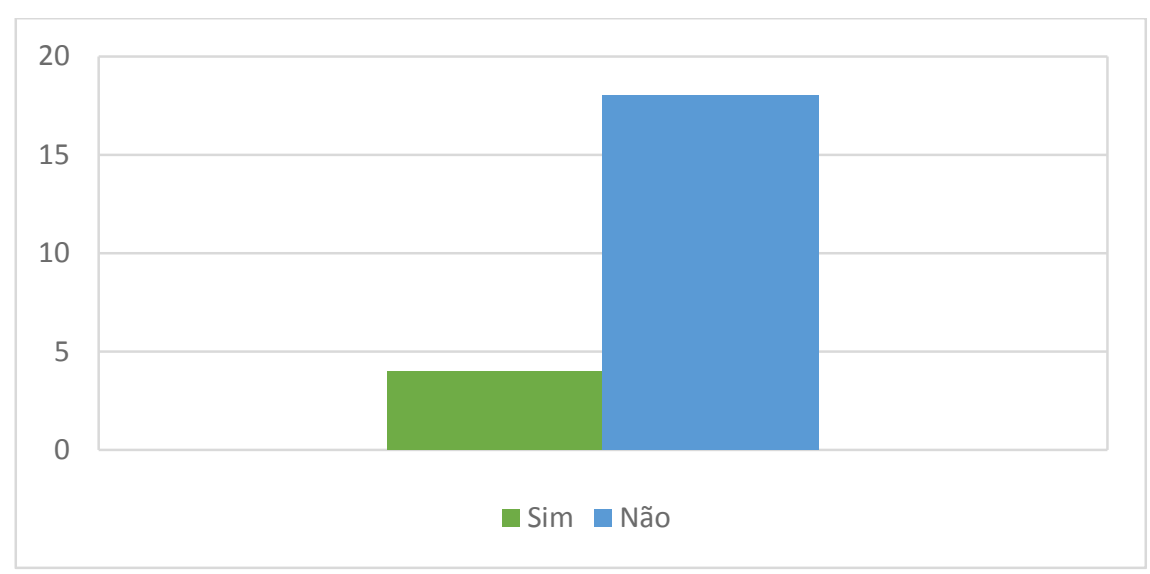

Figura 6: Metodologia utilizada para o plano de reabilitação neuropsicológica.

\section{CONCLUSÃO}

A aplicabilidade da reabilitação neuropsicológica em indivíduos com comprometimento cognitivo decorrente de quadros neurológicos, psiquiátricos, ou de lesões adquiridas já está bem fundamentada na literatura internacional. É cada vez mais frequente a utilização de técnicas e abordagens neuropsicológicas em quadros psicopatológicos complexos, que demandam um trabalho interdisciplinar que inclui psiquiatras, neurologistas, fonoaudiólogos, terapeutas ocupacionais, entre outros. Além disso, a inserção da família e a compreensão do contexto social e cultural do paciente se tornam essenciais para o planejamento de intervenções eficazes.

Por meio da investigação da produção científica sobre reabilitação neuropsicológica no Brasil identificamos que, apesar dos benefícios de sua aplicação para o tratamento de disfunções cognitivas e transtornos neuropsiquiátricos, ainda é reduzido o número de publicações nacionais sobre essa temática. Um dos aspectos positivos encontrados foi que as revistas brasileiras com o maior número de trabalhos publicados são de diferentes áreas do conhecimento, proporcionando, desta maneira, uma perspectiva multiprofissional no desenvolvimento de metodologias em reabilitação neuropsicológica. Apesar disso, identificamos a necessidade de descrição detalhada dos procedimentos de intervenção utilizados nas publicações. Assim, será possível atestar a validade e a confiabilidade dos programas de reabilitação neuropsicológica propostos, a fim de que as técnicas possam ser utilizadas em contextos mais gerais.

Para além desses fatores, constatamos a importância de se estimular a produção de artigos e de aplicações práticas sobre a temática da reabilitação neuropsicológica. Isto possibilitará que sejam realizadas intervenções precoces e, assim, minimizar déficits cognitivos que poderiam interferir na funcionalidade do paciente no seu dia a dia. Acreditamos que o passo inicial seja estabelecer este panorama teórico da reabilitação no nosso país para que a próxima etapa seja efetivamente garantir uma maior aplicabilidade destas técnicas nos indivíduos que delas poderão se beneficiar.

\section{REFERÊNCIAS BIBLIOGRÁFICAS}

CRUZ, S.; SCHEWINSKY, S. R.; ALVES, V. L. R. Implicações das alterações de cognição social no processo de reabilitação global do paciente vítima de traumatismo cranioencefálico. Acta Fisiátr, v. 19, n. 4, p. 207-215, 2012. Disponível em: $<$ http://www.actafisiatrica.org.br/detalhe_artigo.asp?id=479>

FUENTES, D.; MALLOY-DINIZ, L. F.; CAMARGO, C. H. P.; COSENZA, R.M. (eds.). Neuropsicologia: Teoria e Prática. $2^{\mathrm{a}}$ ed. Porto Alegre: Artmed, 2014. 
GINDRI, G.; FRISON, T. B.; OLIVEIRA, C.; ZIMMERMANN, N.; NETTO, T. M.; LANDEIRAFERNANDEZ, J.; PARENTE, M. A. M. P., FONSECA, R. P. Métodos em reabilitação neuropsicológica. Em: J. landeira-Fernandez e Sérgio Fukusima (org), Métodos em Neurociência. São Paulo: Manole, 343 375, 2012.

HAMDAN, A. C.; PEREIRA, A. P. A.; RIECHI, T. I. J. S. Avaliação e Reabilitação Neuropsicológica: Desenvolvimento Histórico e Perspectivas Atuais. Interação em Psicologia, v. 15, número especial, p. 47-58, 2011. Disponível em: <http://revistas.ufpr.br/psicologia/article/view/25373/17001>

HEBBEN, N., \& MILBERG, W. Essentials of neuropsychological assessment. New York: John Wiley \& Sons, 2002.

LEZAK, M. D., HOWIESON, D. B. \& LORING, D. W. Neuropsychological Assessment. (4th ed.). New York: Oxford University Press, 2004.

LURIA, A. R. Fundamentos de Neuropsicologia (J. A. Ricardo, Trad.). São Paulo: Edusp, 1981.

MÄDER, M. J. Avaliação neuropsicológica: aspectos históricos e situação atual. Psicologia: ciência e profissão, $\quad$ v. $\quad 3, \quad$ p. $12-18, \quad 1996 . \quad$ Disponível em: $<$ http://www.scielo.br/scielo.php?script=sci_arttext\&pid=S1414-98931996000300003>

MANCZAK, P. Avanços em neurociências e epilepsia. Instituto Edumed para Educação em Medicina e Saúde. 2003. Disponível em: <www.edumed.org.br/cursos/neurociencia/01/Monografias/neurocienciaepilepsia.doc $>$

rehabilitation of neuropsychological deficits and mild cognitive impairment: A review of the literature. Dementia \& Neuropsychologia, 2(2):139-45, 2008.

PINHEIRO, M. Aspectos históricos da neuropsicologia: subsídios para a formação de educadores. Educar em Revista, 2005. Disponível em :<http://www.redalyc.org/articulo.oa?id=155016226011>

RAMOS, A. A.; HAMDAN, A. C. O crescimento da avaliação neuropsicológica no Brasil: uma revisão sistemática. Psicologia: Ciência e Profissão, v. 36, n. 2, p. 471-485, 2016. Disponível em: $<$ http://www.scielo.br/scielo.php?script=sci_arttext\&pid=S1414-98932016000200471>

RICCIO, C. A.; SUlliVAN, J. R.; COHEN, M. J. Neuropsychological Assessment and Intervention: for Childhood and Adolescent Disorders. New Jersey: Wiley, 2010. 744 p.

SERAPIONI, M. Métodos qualitativos e quantitativos na pesquisa social em saúde: algumas estratégias para a integração. Ciênc. saúde coletiva, v. 5, n. 1, p. 187-192, 2000 . Disponível em: $<$ http://www.scielo.br/scielo.php?pid=S1413-81232000000100016\&script=sci_abstract\&tlng=pt $>$

SIMON, S. S.; RIBEIRO, M. P. O. Comprometimento cognitivo leve e reabilitação neuropsicológica: uma revisão bibliográfica. Psic. Rev., v. 20, n. 1, p. 93-122, 2011. Disponível em: $<$ https://revistas.pucsp.br/index.php/psicorevista/article/viewFile/6795/4918> 